\title{
Dual-Speed MR Safe Pneumatic Stepper Motors
}

\author{
Vincent Groenhuis ${ }^{1}$, Françoise J. Siepel ${ }^{1}$, Stefano Stramigioli ${ }^{1,2}$ \\ ${ }^{1}$ Robotics and Mechatronics, University of Twente, Enschede, The Netherlands \\ ${ }^{2}$ ITMO, Saint Petersburg, Russia \\ Email: v.groenhuis@utwente.nl \\ Supplementary video available at http://www.vincentgroenhuis.nl/DualSpeedMotors.mp4
}

\begin{abstract}
In breast cancer detection it is essential to perform precise interventions to determine the diagnosis. Robotic systems actuated by MR safe pneumatic stepper motors could improve accuracy to target the tumor. The achievable accuracy or speed is limited due to long pneumatic tubes connecting the motors to the electromagnetic valves in the control room. This paper presents the design of two dual-speed stepper motors in order to solve this limitation.

The linear motor measures $50 \times 32 \times 14 \mathrm{~mm}$ (excluding racks) and has step sizes $1.7 \mathrm{~mm}$ and $0.3 \mathrm{~mm}$. The maximum speed under load is $20 \mathrm{~mm} / \mathrm{s}$, measured force is $24 \mathrm{~N}$ and positioning accuracy is $0.1 \mathrm{~mm}$. The rotational motor measures $\varnothing 30 \times 32 \mathrm{~mm}$ (excluding axles) and has step sizes $10^{\circ}$ and $12.9^{\circ}$. Under load its maximum angular speed is $229 \%$ or 38.2 RPM, maximum torque is $74 \mathrm{Nmm}$ and positioning accuracy is $1^{\circ}$. By operating the valves in a coordinated way high-speed and precise position control can be achieved. With these specifications the motors have high potential to actuate MR safe surgical robots.
\end{abstract}

\section{INTRODUCTION}

In breast cancer screening, medical imaging is used by a radiologist to explore abnormalities. When a suspicious lesion is found a biopsy may be required for accurate histological evaluation. In this procedure a biopsy needle is inserted to the location of the suspicious lesion under imaging guidance after which the tissue is sampled and examined. While most lesions are well visible on mammography (x-ray) and ultrasound, a small part is only detectable on MRI. The current MRI-guided breast biopsy procedure is inaccurate and inefficient resulting in long procedure times, additional tissue damage and possibly a false negative biopsy [2, 12].

A robotic system placed inside the MRI scanner allows precise needle operations under near-realtime MRI guidance and could improve the breast biopsy procedure. The construction and actuation of such a robot needs special consideration to avoid interference with the strong magnetic field of the MRI scanner. In the current terminology as defined by the ASTM F2503-13 standard three possible classifications are given to medical equipment: "MR unsafe", "MR conditional" and "MR safe". The strongest classification, "MR safe", is exclusively assigned to devices which are entirely constructed of non-metallic, non-magnetic and non-conductive materials, as determined from its material decomposition rather than MR compatibility tests.

Energy for actuation has to be transferred mechanically to the robot by means of pneumatics, hydraulics or cables. All three methods have its own advantages and disadvantages. A hydraulic actuator utilizes the incompressibility of fluid

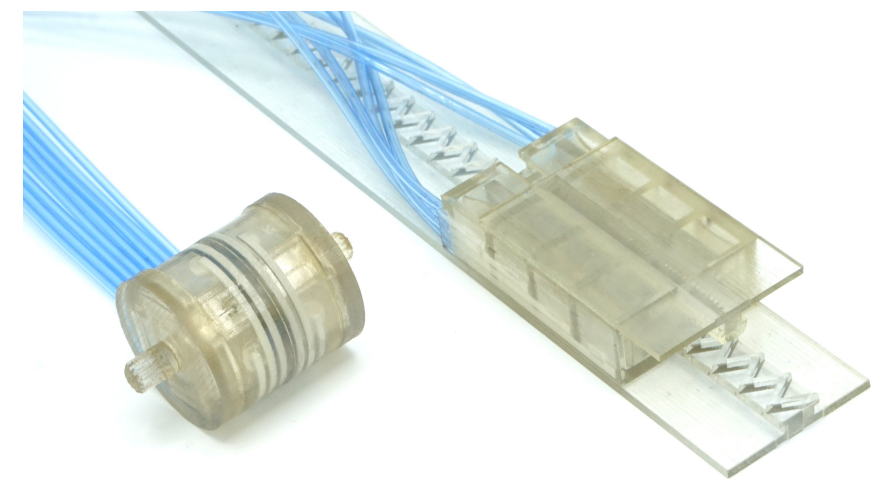

Fig. 1. Photographs of rotational (left) and linear (right) dual-speed pneumatic stepper motors.

to transmit forces and displacements over long distances. Perfect sealing of all parts is required as leaking liquid is troublesome [13], making it difficult to produce parts by rapid prototyping techniques. Actuation by Bowden cables and/or pulley systems have been proven effective in many areas of research and engineering: forces and displacements are transferred by pushing, pulling and/or twisting a cable inside the tube. The main challenge involved is to overcome the static friction, especially when the cable has numerous bends between the robot and controller [8]. Pneumatic systems utilize pressurized air as the medium which has the advantages that it is abundant in hospitals, small leaks are acceptable and long transmission distances are possible without significant drop in supplied pressure. An important drawback is that air is compressible: while a single pneumatic cylinder could be used as an actuator [3], precise position control of the piston is difficult and the only well-defined positions are the two end-stop positions. The air compressibility issue can be mitigated by utilizing the stepper motor mechanism: two or more pneumatic cylinders drive a rack or gear in discrete steps, resulting in a pneumatic stepper motor [4, 7, 9] on which this research is based.

The relevant characteristics of an MR safe motor are size, force, accuracy and speed. The motor must fit within a device alongside the patient inside the MRI scanner, so the size must be in the order of a few centimetres. The maximum force must be at least $20 \mathrm{~N}$ to allow inserting a biopsy needle 
in dense tissue. The speed should be sufficient to move the end-effector to a specified location within approximately ten seconds in order not to delay the biopsy procedure. Finally, sub-millimeter accuracy and precision are required to allow targeting of millimeter-sized lesions.

\section{A. State-of-art}

Several MR safe robotic systems driven by pneumatic stepper motors have been demonstrated in literature. Stoianovici et al. [10] demonstrated the MrBot driven by PneuStep motors, a rotational stepper motor with three phases and optical position feedback. The time needed to align the needle guide with the target site is $1: 36 \pm 1.24 \mathrm{~min}$ [11]. Bomers et al. [1] developed the RCM (remote-controlled manipulator), a robotic system for transrectal prostate biopsy and driven by helical pneumatic stepper motors. The mean manipulation time for needle guide movement was measured to be $5: 48 \mathrm{~min}$. Groenhuis et al. [7] developed the Stormram 4, which requires up to $1: 30 \mathrm{~min}$ to manipulate the needle to the commanded position.

All these robots require more than one minute to move to the target position, delaying the biopsy procedure. The low speed is a result of the sub-millimeter step sizes combined with low stepping frequencies limited by the $5 \mathrm{~m}$ to $10 \mathrm{~m}$ tubes that connect the motors to MR-unsafe valve manifolds outside the Faraday cage of the MRI scanner.

\section{B. Analysis of maximum stepping frequency}

The maximum frequency of a pneumatic stepper motor is primarily limited by tube dimensions and valve specifications. In order to move a piston pneumatically one chamber has to be pressurized and the opposite chamber (if any) de-pressurized, both involving the displacement of a certain volume of air through the valve. As the internal volume of a pneumatic tube ( $24.5 \mathrm{~cm}^{3}$ for $5 \mathrm{~m}$ long, $2.5 \mathrm{~mm}$ inner diameter tubes) is an order of magnitude higher than the cylinder stroke of a stepper motor (order of $1 \mathrm{~cm}^{3}$ ), the tube also has characterstics of a reservoir and the airflow is not constant across the length of the tube, complicating theoretical analysis.

Two different valves are considered. The MHP2 (Festo MHP2-MS1H-5/2-M5, Festo AG \& Co. KG, Esslingen am Neckar, Germany) is a direct-acting solenoid valve with a specified switching time of $2 \mathrm{~ms}$. The PV5211 (PneumatiekVoordeel PV5211-24VDC-1/8, TechniComponents B.V., Waalwijk, The Netherlands) is an internally-piloted valve with a specified switching time of under $50 \mathrm{~ms}$. In the PV5211 valve the delay between the switching of the pilot and main valves does not necessarily affect the rise time of the valve, instead it primarily contributes to a phase shift in its frequency response.

The flow rate is restricted by the $2.5 \mathrm{~mm}$ orifice diameters of the valve and tubes. This results in a theoretical maximum flow rate of $343 \pi \times 0.00125^{2} \mathrm{~m}^{3} / \mathrm{s}=1680 \mathrm{~cm}^{3} / \mathrm{s}=101 \mathrm{~L} \mathrm{~min}^{-1}$. The specified nominal flow rates for both valves is approximately $90 \mathrm{~L} \mathrm{~min}^{-1}=1500 \mathrm{~cm}^{3} / \mathrm{s}$ for a system pressure of $0.3 \mathrm{MPa}$ to $0.5 \mathrm{MPa}$ and a pressure drop of $0.1 \mathrm{MPa}$ across the valve, which are consistent with the calculated maximum flow rates.

Ignoring switching time of the valve the minimum time needed to pressurize a volume of $25 \mathrm{~cm}^{3}$ is $\frac{25}{1500} \mathrm{~s} \approx 17 \mathrm{~ms}$. This lower bound cannot be significantly reduced by using differently-sized valves or tubes, as the minimum travel time for a pocket of air through a $5 \mathrm{~m}$ tube is $\frac{5}{343} \mathrm{~s}=15 \mathrm{~ms}$. This sets a strict upper bound of $\frac{1}{0.015} \mathrm{~Hz}=67 \mathrm{~Hz}$ for the stepping frequency. The actual bandwidth is lower due to friction and dynamics in the system.

Yang et al. [14] approximated the dynamics of a $9 \mathrm{~m}$ long, $3.2 \mathrm{~mm}$ inner diameter tube as a first-order system with bandwidth $5.1 \mathrm{~Hz}$ and time delay $27 \mathrm{~ms}$. Groenhuis and Stramigioli [5] experimentally observed a bandwidth of $10 \mathrm{~Hz}$ when using $5 \mathrm{~m}$ long, $2.5 \mathrm{~mm}$ inner diameter tubes. Higher stepping frequencies are accompanied with a loss of force or torque. Stoianovici et al. [10] implemented position feedback using optical waveguides in order to optimize the stepping frequency for a given load and also account for missing steps.

\section{Approach}

The aim of this paper is to achieve higher accuracy and speed in pneumatic stepper actuators suitable for MR safe surgical robots. The approach is to combine two singular stepper motors with different step sizes into one housing in a space-efficient way. By simultaneously operating both the large-step side and the small-step side in constructive directions, maximum speed is achieved which is the sum of the individual speeds. Smaller steps can be made by operating the small-step side only or by operating both sides in opposite directions. Even smaller net displacements may be achieved by performing a combination of multiple large and small steps. No position feedback is employed to keep the design as compact and simple as possible. Instead, correctness of controlled position is guaranteed when the motor is not overloaded.

One linear and one rotational dual-speed stepper motor have been developed to demonstrate the different geometries and design parameters and also provide solutions for different types of kinematic joints. The linear motor actuates a prismatic joint while the rotational motor can directly actuate a revolute joint. The step sizes in the linear motor are substantially different while the step sizes of the rotational motor are close to each other.

\section{Materials And Methods}

\section{A. Linear motor design}

Figure 2 shows an exploded view of the dual-speed linear motor. It is based on the design by Groenhuis et al. [6, 7] and consists of a housing (red) with four dual-acting pneumatic cylinders (green) sealed by $10 \times 10 \mathrm{~mm}$ square-shaped seals (yellow) acting on two racks (purple). The large-step side consisting of the outermost two cylinders drives the long rack on the bottom which has a teeth pitch of $6.8 \mathrm{~mm}$ and a teeth depth of $6 \mathrm{~mm}$. The small-step side consisting of the innermost two cylinders drives the short rack on the top which has a teeth 


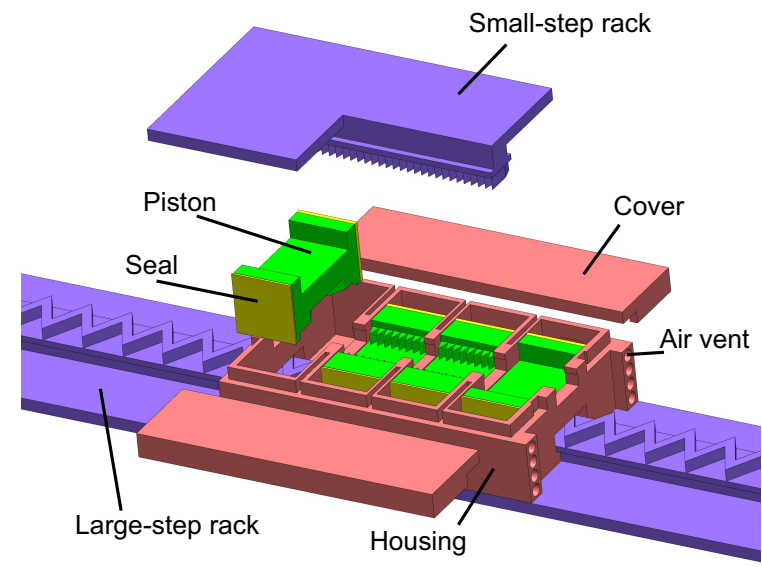

Fig. 2. Exploded view of linear motor. The pistons (green) slide back and forth inside the box-shaped cavities of the housing (red) sealed by the square seals (yellow). The two outermost pistons have large teeth on the bottom, driving the large-step rack (purple). The two innermost pistons actuate the small-step rack on top.

pitch of $1.2 \mathrm{~mm}$ and a teeth depth of $2 \mathrm{~mm}$. The linear stepper motor measures $50 \times 32 \times 14 \mathrm{~mm}$ excluding racks.

The force exerted by one piston at a pressure of $0.3 \mathrm{MPa}$ is $0.3 \times 10^{6} \times 0.01^{2} \mathrm{~N}=30 \mathrm{~N}$. The teeth in the largestep side have a mechanical advantage of $\frac{2 \times 6}{6.8}=1.76$ so the force exerted by the large-step rack (ignoring friction losses) is $1.76 \times 30 \mathrm{~N}=53 \mathrm{~N}$. For the small-step side the advantage ratio is $\frac{2 \times 2}{1.2}=3.33$ resulting in a theoretical force of $3.33 \times 30 \mathrm{~N}=100 \mathrm{~N}$.

The range of motion is determined by the length of the longer rack. The shorter rack is primarily used to enhance positioning accuracy. As 17 small steps result in the same displacement $(5.4 \mathrm{~mm})$ as three large steps, the minimum range of motion for the small-step rack to reach all positions is $5.4 \mathrm{~mm}$. Excess range may still be useful for reaching the desired target position in a lower number of steps.

\section{B. Rotational motor design}

Figure 3 shows an exploded view of the dual-speed rotational motor. The number and cross-sectional area of the cylinders are identical to that of the linear motor, but these are placed in a cross configuration and the teeth act on geared axles instead of racks. The axle of the large-step side has seven teeth resulting in a step angle of $\frac{90}{7}=12.86^{\circ}=0.224 \mathrm{rad}$, while the small-step axle has nine teeth and a step angle of $10^{\circ}=0.175 \mathrm{rad}$. The teeth depth on both sides is $2.5 \mathrm{~mm}$. Excluding axles, the motor dimensions are $\varnothing 30 \mathrm{~mm} \times 32 \mathrm{~mm}$.

The piston force at a pressure of $0.3 \mathrm{MPa}$ is also $30 \mathrm{~N}$. For the large-step side this results in a theoretical torque of $\frac{30 \times 0.0025}{2 \times 0.224} \mathrm{Nm}=167 \mathrm{~N} \mathrm{~mm}$, while for the small-step side the theoretical torque is $\frac{30 \times 0.0025}{2 \times 0.175} \mathrm{~N} \mathrm{~m}=214 \mathrm{~N} \mathrm{~mm}$.

In a typical application one of the axles is fixed to an inertial reference frame. Actuation of this side will cause the housing to revolve around the axle. The range of motion of this side is constrained by the amount of available space to

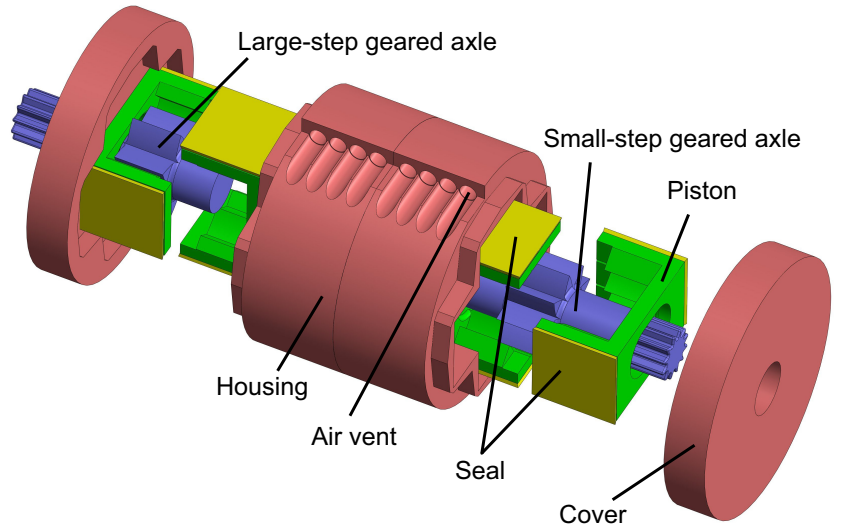

Fig. 3. Exploded view of rotational motor. The pistons (green) act on the geared axles (purple). The large-step axle gear (left) has seven teeth and takes 28 steps per revolution. The small-step axle gear (right) has nine teeth, taking 36 steps per revolution.

wrap and un-wrap the pneumatic tubes, which must be at least $90^{\circ}$ to be able to reach all setpoints. The other side can rotate continuously if not constrained by the range of motion of the actuated kinematic joint.

Figure 4 shows a schematic cross-sectional view of the large-step rotational unit through a sequence of five states. The two pistons (red and green) both make contact with the geared axle (purple), but only the piston which was pressurized formerly establishes full contact with the gear and determines the position. This eliminates backlash but also causes hysteresis when the direction is reversed. When the four cylinder chambers are pressurized in a particular sequence the gear performs step-wise rotational movements in the desired direction. Figure 5 shows a similar operating sequence for a generic linear stepper motor unit.

\section{Production}

Figure 11 shows both assembled motors. The rigid parts of both motors were printed on a Stratasys Connex 3 printer (Stratasys, Ltd., Eden Prairie, MN, USA) in VeroClear material, standard quality, glossy finish. The seals were laser-cut from $0.5 \mathrm{~mm}$ silicone rubber. The moving parts were lubricated with petroleum jelly (vaseline) and the housing was glued together using Loctite 406. Polyurethane tubing with an inner diameter of $2.5 \mathrm{~mm}$ and a length of $5 \mathrm{~m}$ was used to connect the motors to the valves. All used materials are non-metallic, non-magnetic and non-conductive.

\section{Position control}

The linear actuator can perform displacements of $0.3 \mathrm{~mm}$, $1.4 \mathrm{~mm}, 1.7 \mathrm{~mm}$ or $2.0 \mathrm{~mm}$ in either direction within a single step. The greatest common divisor of the available step sizes is $0.1 \mathrm{~mm}$, which is the discretization error when executing an arbitrary number of steps.

Given a target displacement $d$, the number of large steps $m$ and small steps $n$ need to be calculated such that $1.7 m+0.3 n=d$. The range of motion of both sides gives 

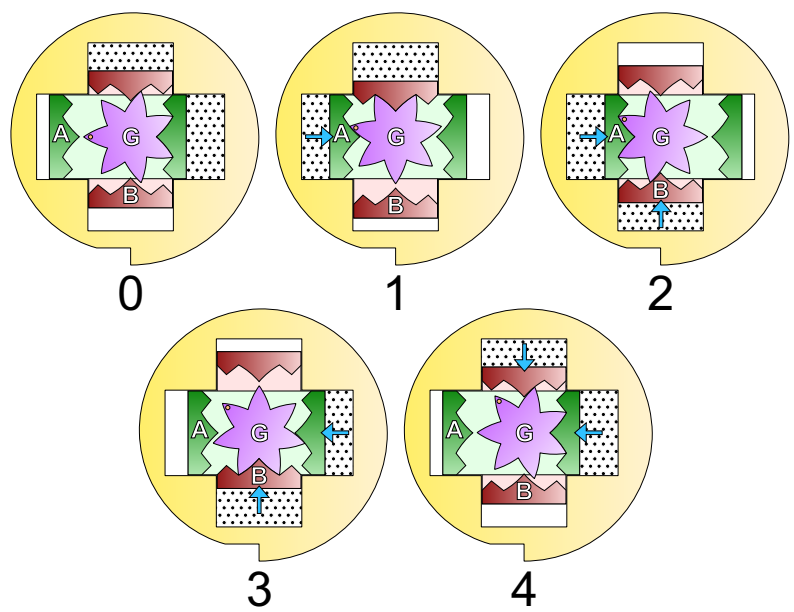

Fig. 4. Five consecutive states of the large-step side of the rotational motor with the housing (yellow), geared axle (purple) and pistons (red and green). A step is performed by pneumatically moving one piston to the opposite side. After performing four steps the gear has rotated by one-seventh revolution.
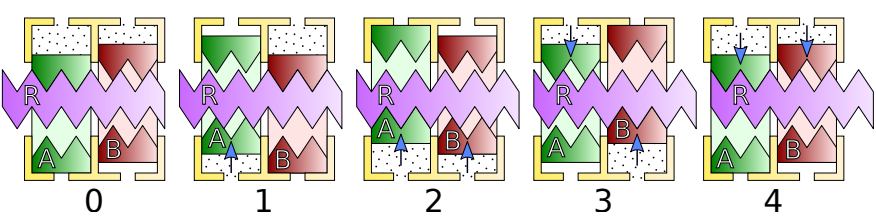

Fig. 5. Five consecutive states of a single-speed linear stepper motor with the housing (yellow), rack (purple) and pistons (red and green).

an additional constraint and the total number of steps, $\max (|m|,|n|)$, is to be minimized for efficient operation. The lower bound for the required number of steps is $\left\lceil\frac{d}{2.0}\right\rceil$ and an upper bound is $\left\lceil\frac{d}{1.7}+20\right\rceil$. The optimal values for $m$ and $n$ can be found with an iterative algorithm or an exhaustive search over the whole state space.

For the rotational actuator the possible displacements in a single step are $2.86^{\circ}, 10^{\circ}, 12.86^{\circ}$ and $22.86^{\circ}$. The greatest common divisor is $1.43^{\circ}$ and the required number of steps to perform a target angular displacement can be found using the same methods as for the linear stepper motor.

The execution of large and small steps in opposite directions may cause overshooting, for example when a net displacement of $0.1 \mathrm{~mm}$ has to be executed. If overshoot is undesired, the precision of small adjustments changes to $0.3 \mathrm{~mm}$ which still complies with the sub-millimeter accuracy requirement. It is also possible to avoid overshooting in one particular direction only (e.g. the forward direction) by operating the motor sides in a certain sequence such that all backward motion(s) are performed prior to all forward motion(s). In that situation, any overshoot only occurs in the backward direction.

To reduce the hysteresis effect, setpoints for both motor sides should be consistently approached in forward direction. If a setpoint was approached in backward direction, the associated hysteresis effect can be cancelled by executing reciprocal steps.

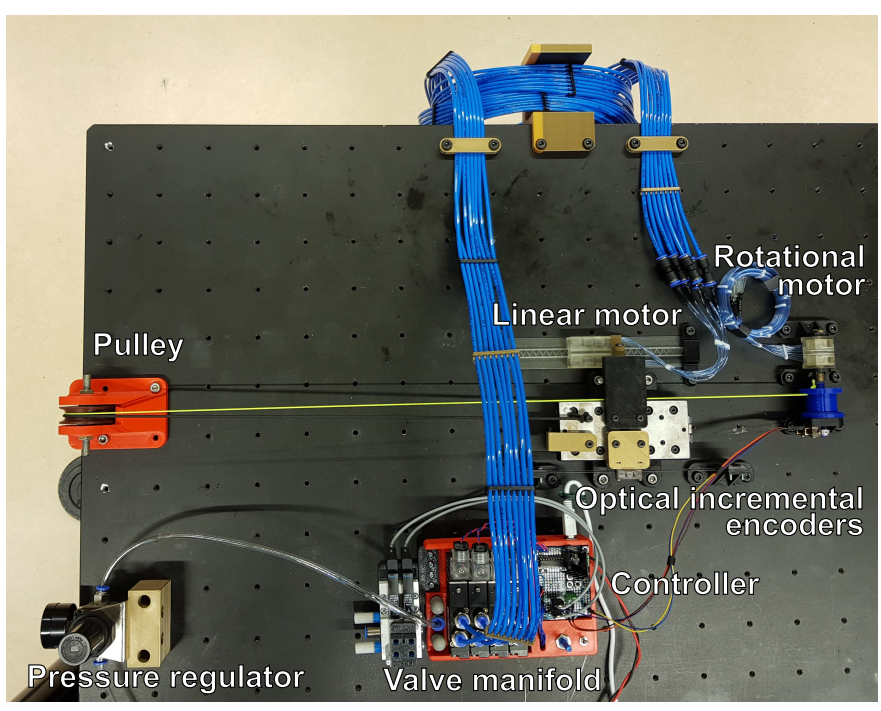

Fig. 6. Measurement setup for linear and rotational motors. The motor's outputs are connected to weights of known mass hanging over a pulley. The pressure regulator can be adjusted to find the minimum pressure required for the motor to lift the given weights. Optical encoders track the precise displacements of the motors.

\section{EVALUATION}

To study the transient behaviour, positional accuracy, speed and maximum force/torque of the motors, several experiments were performed.

\section{A. Measurement setup}

Figure 6 shows the measurement setup for the linear and rotational motors. The linear motor is attached to a sliding rail of which the position is measured with an optical encoder of type EM2-0-1000-I (US Digital, Vancouver, WA, USA) with a resolution of $0.00635 \mathrm{~mm}$. Forces on the slider are generated by bodies with known mass hanging on a pulley.

The rotational motor drives a spindle with radius $15 \mathrm{~mm}$ to lift bodies of known mass. The angular displacement is measured using an optical encoder of type HEDS-9140 (Broadcom Ltd., San Jose, CA, USA) with a resolution of $0.18^{\circ}$.

Polyurethane tubes (outer diameter $4 \mathrm{~mm}$, inner diameter $2.5 \mathrm{~mm}$, length $5 \mathrm{~m}$ ) connect either motor to the valves of type PV5211-24VDC-1/8. The system pressure is adjusted by a manual pressure regulator and observed by a pressure gauge.

An Arduino controls the valves at a fixed frequency of $10 \mathrm{~Hz}$ in all tests while keeping track of the optically-encoded position of the motor.

\section{B. Experiments}

1) Positional accuracy: In the positional accuracy experiments, the displacements of both motors were recorded while navigating through a sequence of setpoints. All positions were zero-calibrated at $\mathrm{t}=0 \mathrm{~s}$. The transient displacement was studied for overshoot and delay characteristics. The offset between setpoint and measured steady-state position resulted in a measure of positional accuracy. 


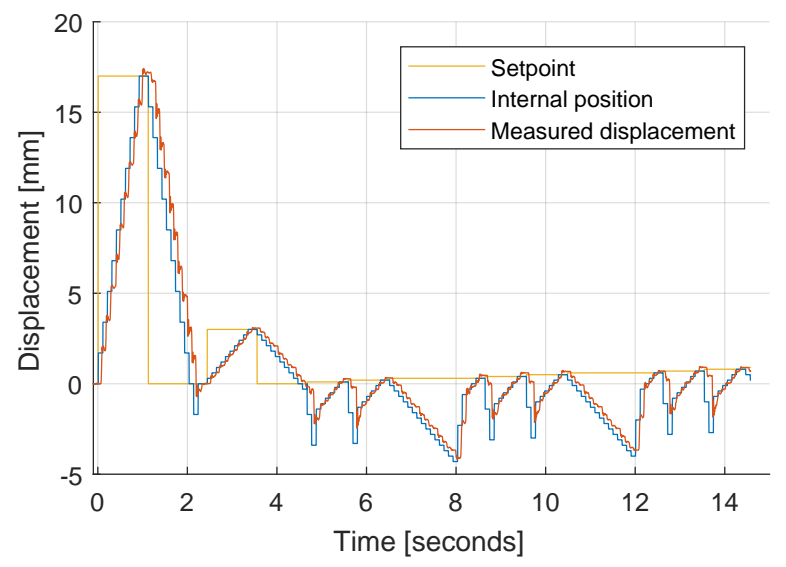

Fig. 7. Transient displacement of linear motor. In the first two seconds the large-step side $(1.7 \mathrm{~mm})$ was operated, followed by operation of the smallstep side $(0.3 \mathrm{~mm})$. Afterwards a series of net displacements of $0.1 \mathrm{~mm}$ were executed by driving combinations of large and small steps in such a way that no overshooting occurs in forward direction.

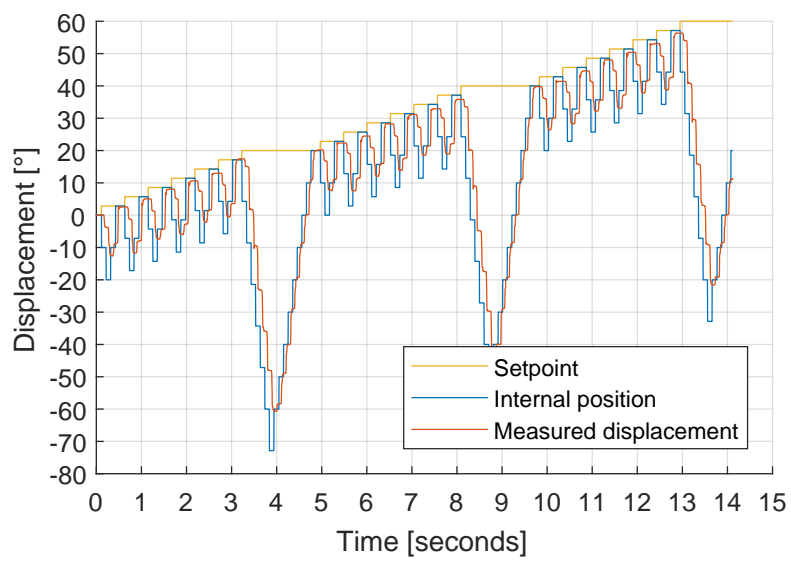

Fig. 8. Transient displacement of rotational motor. A series of $2.86^{\circ}$ steps were executed by rotating the two sides in opposite directions. Certain additional steps were necessary to take hysteresis and range constraints into account.

2) Force and torque: The linear motor was loaded using bodies with a range of masses in steps of $0.5 \mathrm{~kg}$. For each load the lowest pressure was determined such that the largestep side of the motor was just able to lift the applied weight without skipping steps at a frequency of $10 \mathrm{~Hz}$. The same procedure was performed for the small-step side of the motor.

The rotational motor was tested using the same setup, but its spindle was loaded with a series of masses in steps of $0.1 \mathrm{~kg}$.

\section{Results}

1) Transient displacement: Figure 7 shows the transient displacement of the linear motor. From $\mathrm{t}=0 \mathrm{~s}$ it executes ten large steps, travelling $17 \mathrm{~mm}$ within one second. The average measured propagation delay was $70 \mathrm{~ms}$. In the next second it travels back to $\mathrm{x}=0 \mathrm{~mm}$. The deviation between internal and measured position is corrected by the extra reciprocating step at $\mathrm{t}=2.2 \mathrm{~s}$. From $\mathrm{t}=2 \mathrm{~s}$ it executes ten small steps to

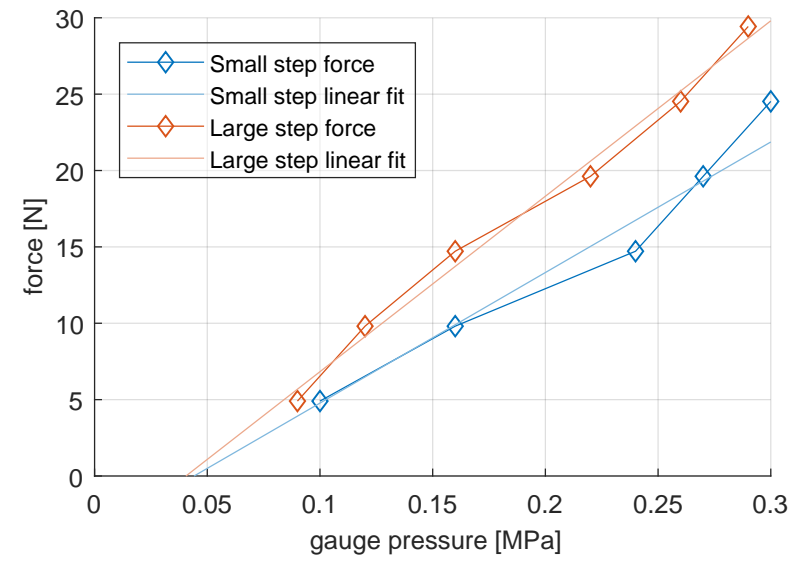

Fig. 9. Maximum forces of the linear motor for a range of pressures, for both motor sides.

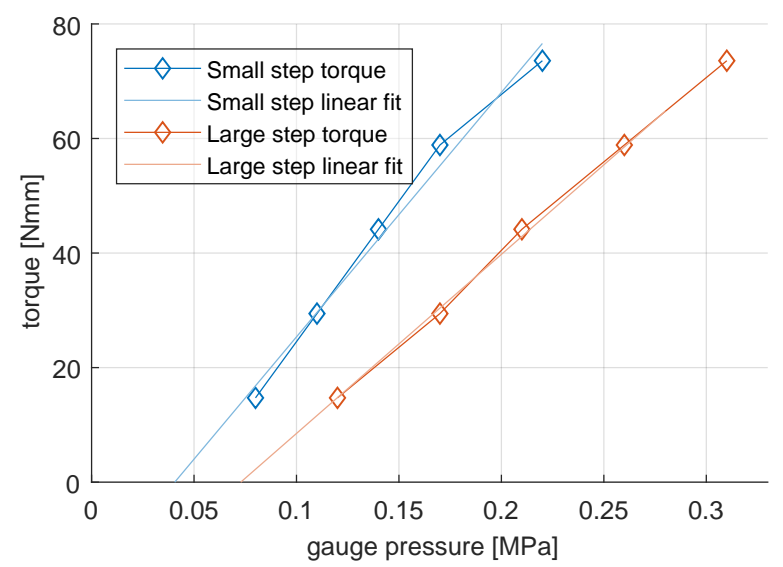

Fig. 10. Maximum torques of rotational motor for a range of pressures, for both motor sides.

$\mathrm{x}=3.0 \mathrm{~mm}$ and back to the origin. From there on the setpoint is incremented in steps of $0.1 \mathrm{~mm}$ and it can be seen that for every $0.1 \mathrm{~mm}$ net displacement the motor executes a number of backward steps followed by a number of forward steps. Upon reaching the setpoint in forward direction the motor overshoots by less than $0.1 \mathrm{~mm}$ and the steady-state error is $0.1 \mathrm{~mm}$ on average. The recorded movements can also be viewed in the supplementary video.

Figure 8 shows the transient angular displacement of the rotational motor. The setpoint position is increased in steps of $2.86^{\circ}$ and it can be observed that in most cases four steps are required. The small-step side first executes two backward steps followed by one forward step after which the large-step side executes one forward step. More steps are executed around $\mathrm{t}=4 \mathrm{~s}$ and $\mathrm{t}=9 \mathrm{~s}$ to avoid wrapping the pneumatic tubes, hereby rotating at $100 \%$ to $128 \%$ s. During forward motion of the motor sides, it follows the internal setpoint with a time delay of approximately $60 \mathrm{~ms}$. Upon reaching each setpoint the error between setpoint and observed position was measured to be $-0.69 \pm 0.52^{\circ}$. 


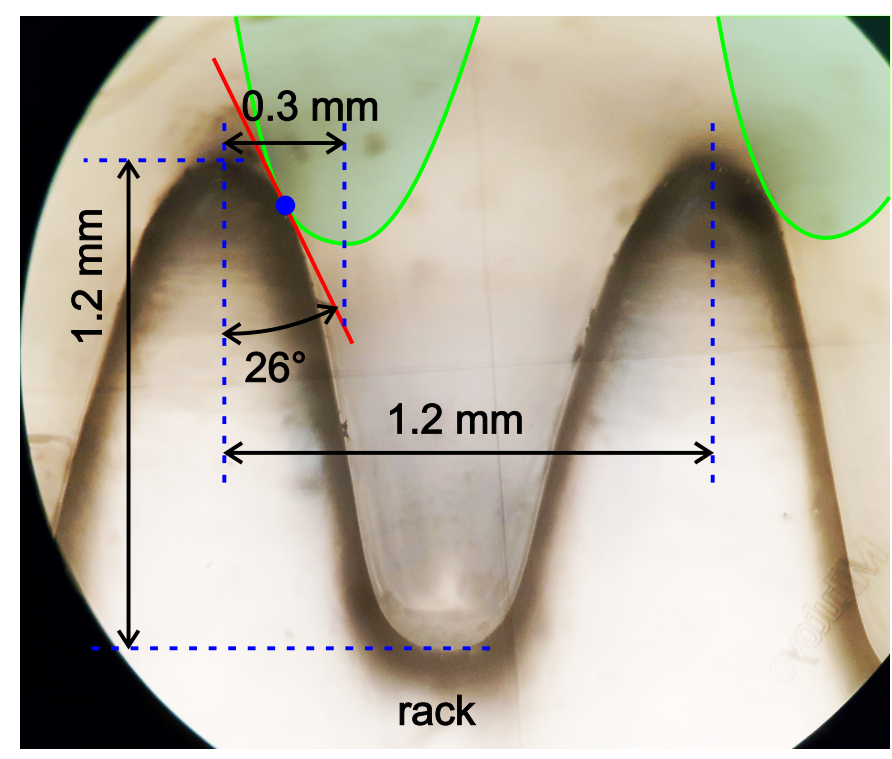

Fig. 11. Microscopic picture of the small-step rack showing the roundedness of its teeth. One point of contact with the engaging piston teeth (sketched green) is shown as a blue dot and its tangent line in red. The slope of this tangent line determines the effective wedge ratio of the mechanical transfer from piston to rack.

2) Force and torque: Figure 9 shows the maximum force of both sides of the linear motor at different pressures. At a pressure of $0.3 \mathrm{MPa}$ the small-step side delivers $24 \mathrm{~N}$ which means an efficiency of $\frac{24}{100}=24 \%$, while the large-step rack exerts $29 \mathrm{~N}$ resulting in an efficiency of $\frac{29}{53}=55 \%$. Examination of the teeth shape under a microscope (Figure 11) revealed that the small-step teeth are rounded due to 3D printing effects and that the actual teeth depth is $1.2 \mathrm{~mm}$ instead the designed $2.0 \mathrm{~mm}$, reducing the effectiveness of the wedge mechanism. When engaged by the piston teeth, the effective wedge ratio at the contact point is $\frac{1}{\tan 26^{\circ}}=2.05$ and the difference with the theoretical value of 3.33 makes up for the majority of the difference in observed mechanical effiency between both sides of the linear motor.

In case of operating the motor under a $24 \mathrm{~N}$ load the maximum combined work per step is $24 \mathrm{~N} \times(0.3+1.7) \mathrm{mm}=$ $48 \mathrm{~mJ}$. At an operating frequency of $10 \mathrm{~Hz}$ this results in a maximum power of $0.48 \mathrm{~W}$.

Figure 10 shows the maximum torques for the rotational motor. At a pressure of $0.3 \mathrm{MPa}$ the torque of the large-step side was measured to be $74 \mathrm{Nmm}$, resulting in a mechanical efficiency of $\frac{74}{167}=44 \%$. The small-step side is able to exert the same torque at a pressure of $0.22 \mathrm{MPa}$, resulting in a mechanical efficiency of $\frac{74}{0.733 \times 214}=47 \%$.

The combined work per step under a $72 \mathrm{Nmm}$ load is $72 \mathrm{~N} \mathrm{~mm} \times(0.224+0.175)=29 \mathrm{~mJ}$. At a stepping frequency of $10 \mathrm{~Hz}$ this gives a maximum power of $0.29 \mathrm{~W}$.

\section{Discussion}

The linear motor has shown that it can move with a speed of $20 \mathrm{~mm} / \mathrm{s}$, has a minimum step size of $0.3 \mathrm{~mm}$, a positioning accuracy of $0.1 \mathrm{~mm}$ and a maximum force of $24 \mathrm{~N}$ at a pressure of $0.3 \mathrm{MPa}$. A displacement of $100 \mathrm{~mm}$ can be performed by executing 56 large and 16 small steps which takes $5.6 \mathrm{~s}$ in total. This is an order of magnitude faster than state-of-the-art stepper motors, without compromising on force or accuracy.

The rotational motor can rotate at a speed of up to $229 \%$, achieve an accuracy of approximately $1^{\circ}$, has a minimum step size of $2.86^{\circ}$ (when operating both sides in opposite direction) and delivers a maximum torque of $74 \mathrm{Nmm}$ at $0.3 \mathrm{MPa}$. A rack-and-pinion with radius $5 \mathrm{~mm}$ theoretically converts these figures to a linear speed of up to $20 \mathrm{~mm} / \mathrm{s}$ with an accuracy of $0.09 \mathrm{~mm}$ and a maximum force of $15 \mathrm{~N}$.

The maximum forces and torques are limited by system pressure, cylinder cross-sectional area and the effective wedge ratio of the mechanical force transfer from piston to rack or gear. A larger cylinder cross-sectional area would result in enlarged overall dimensions, while increased pressure or a higher wedge ratio require the use of stronger materials to withstand the increased material stresses. In the linear motor the force is also limited by the roundedness of the teeth in the small-step rack due to manufacturing limitations, resulting in a suboptimal wedge mechanism.

In the rotational motor the small-step side requires $30 \%$ lower pressure to deliver the same amount of torque than the large-step side. The reason is that the step angle is different while the teeth depth is equal, resulting in inconsistent wedge ratios. A performance gain can be expected by better balancing the maximum torques of both sides, which could be achieved by adjusting the dimensions of the teeth or cylinders while taking the size and material strength constraints into account.

The range of motion of the linear actuator depends on the lengths of the large-step and small-step racks. The large-step rack must be long enough to cover the entire workspace, while the small-step rack only needs sufficient freedom to make the required small-step adjustements. The small-step side can move continuously in the rotational motor while the large-step side is constrained by the available space to wrap and un-wrap the pneumatic tubes.

The bandwidth is limited to $10 \mathrm{~Hz}$, so higher stepping frequencies result in reduced output forces. Steps may be skipped when load forces exceed the motor output forces and these skipped steps cannot be immediately detected without position feedback. While it is possible to incorporate MR safe position feedback with optical sensors, a possibly more viable approach to further increase travel speed is to add a third stage resulting in a triple-speed pneumatic stepper motor.

Eight pneumatic tubes with outer diameter $3 \mathrm{~mm}$ to $4 \mathrm{~mm}$ are needed to connect one dual-speed motor to four valves of the controller, resulting in a relatively large cable bundle and complex controller for the whole robotic system. The number of tubes may be reduced by employing cylinder sharing techniques, at the cost of a reduced state space.

The size, force, accuracy and speed characteristics of both dual-speed stepper motors make these suitable for effective actuation of MR safe robotic systems. In particular, the linear motor could be used in the linear stages of the Stormram 4 robot [7]. Based on the modified joint velocities the projected 
TABLE I

AVERAGE MOVEMENT TIME OF BIOPSY ROBOTS

\begin{tabular}{c|c} 
Robotic system & Operating time (minutes) \\
\hline Stoianovici et al. (MrBot) [11] & $1: 36 \pm 1: 24$ \\
Bomers et al. (RCM) [1] & $5: 48$ \\
Groenhuis et al. (Stormram 4) [7] & $1: 30$ \\
System using dual-speed linear motors & $0: 11$ (projected)
\end{tabular}

average time required to move the upgraded robot towards a new target location can be calculated. A typical movement involves a displacement over $50 \mathrm{~mm}$ (28 steps of $1.7 \mathrm{~mm}$ plus 8 steps of $0.3 \mathrm{~mm}$ ) followed by an angulation of $10^{\circ}$ (40 steps of $0.25^{\circ}$ ) and the insertion of a biopsy needle over a distance of $70 \mathrm{~mm}$ (41 steps of $1.7 \mathrm{~mm}$ plus one of $0.3 \mathrm{~mm}$ ). In total this requires 109 steps which can be executed in $10.9 \mathrm{~s}$ at a stepping frequency of $10 \mathrm{~Hz}$. For comparison, Table $\mathrm{I}$ lists the average movement durations for different MR safe biopsy robots driven by pneumatic stepper motors.

\section{CONCLUSiON}

Two space-efficient dual-speed motors have been developed. Both of them are one order of magnitude faster than stateof-art pneumatic stepper motors, without compromising on accuracy or force. Limitations include the high number of pneumatic tubes and the additional complexity involved in manufacturing and control, especially when enhanced accuracy without overshooting is desired. The average required time to manipulate the robot using such motors to a given position can be reduced from minutes to approximately ten seconds. This improves the potential for MR safe robotic devices to improve accuracy and reduce the procedure time of a MR guided breast biopsy.

\section{ACKNOWLEDGEMENTS}

The authors thank Gerben te Riet o/g Scholten for the support in the production process of the motors.

\section{REFERENCES}

[1] J. G. R. Bomers, D. G. H. Bosboom, G. H. Tigelaar, J. Sabisch, J. J. Fütterer, and D. Yakar. Feasibility of a 2nd generation MR-compatible manipulator for transrectal prostate biopsy guidance. European Radiology, 27(4):1776-1782, 2017. ISSN 14321084. doi: 10.1007/s00330-016-4504-2. URL http://dx.doi.org/10. 1007/s00330-016-4504-2.

[2] M. C. Chevrier, J. David, M. E. Khoury, L. Lalonde, M. Labelle, and I. Trop. Breast Biopsies Under Magnetic Resonance Imaging Guidance: Challenges of an Essential but Imperfect Technique. Curr Probl Diagn Radiol, 45 (3):193-204, 2016. doi: 10.1067/j.cpradiol.2015.07.002. URL https://doi.org/10.1067/j.cpradiol.2015.07.002

[3] E. Franco, D. Brujic, M. Rea, W. M. Gedroyc, and M. Ristic. Needle-Guiding Robot for Laser Ablation of Liver Tumors Under MRI Guidance. IEEE/ASME Transactions on Mechatronics, 21(2):931-944, April 2016. ISSN 1083-4435. doi: 10.1109/TMECH.2015.2476556. URL https://doi.org/10.1109/TMECH.2015.2476556.
[4] V. Groenhuis and S. Stramigioli. Laser-Cutting Pneumatics. IEEE/ASME Transactions on Mechatronics, 21 (3):1604-1611, June 2016. ISSN 1083-4435. doi: 10.1109/TMECH.2015.2508100. URL https://doi.org/10. 1109/TMECH.2015.2508100.

[5] V. Groenhuis and S. Stramigioli. Rapid Prototyping High-Performance MR Safe Pneumatic Stepper Motors. IEEE/ASME Transactions on Mechatronics, page In press, 2018. doi: 10.1109/TMECH.2018.2840682. URL https://doi.org/10.1109/TMECH.2018.2840682.

[6] V. Groenhuis, J. Veltman, F. J. Siepel, and S. Stramigioli. Stormram 3: A Magnetic Resonance ImagingCompatible Robotic System for Breast Biopsy. IEEE Robotics Automation Magazine, 24(2):34-41, June 2017. ISSN 1070-9932. doi: 10.1109/MRA.2017.2680541. URL https://doi.org/10.1109/MRA.2017.2680541.

[7] V. Groenhuis, F. J. Siepel, J. Veltman, J. K. van Zandwijk, and S. Stramigioli. Stormram 4: An MR Safe Robotic System for Breast Biopsy. Annals of Biomedical Engineering, May 2018. ISSN 1573-9686. doi: 10. 1007/s10439-018-2051-5. URL https://doi.org/10.1007/ s10439-018-2051-5.

[8] N. Hungr, I. Bricault, P. Cinquin, and C. Fouard. Design and Validation of a CT- and MRI-Guided Robot for Percutaneous Needle Procedures. IEEE Transactions on Robotics, 32(4):973-987, Aug 2016. ISSN 15523098. doi: 10.1109/TRO.2016.2588884. URL https: //doi.org/10.1109/TRO.2016.2588884

[9] H. Sajima, H. Kamiuchi, K. Kuwana, T. Dohi, and K. Masamune. MR-Safe Pneumatic Rotation Stepping Actuator. Journal of Robotics and Mechatronics, 24(5): 820-827, 2012. doi: 10.20965/jrm.2012.p0820. URL https://doi.org/10.20965/jrm.2012.p0820.

[10] D. Stoianovici, A. Patriciu, D. Petrisor, D. Mazilu, and L. Kavoussi. A New Type of Motor: Pneumatic Step Motor. IEEE/ASME Transactions on Mechatronics, 12(1):98-106, Feb 2007. ISSN 1083-4435. doi: 10.1109/TMECH.2006.886258. URL https://doi.org/10. 1109/TMECH.2006.886258.

[11] D. Stoianovici, C. Kim, D. Petrisor, C. Jun, S. Lim, M. W. Ball, A. Ross, K. J. Macura, and M. E. Allaf. MR Safe Robot, FDA Clearance, Safety and Feasibility of Prostate Biopsy Clinical Trial. IEEE/ASME Transactions on Mechatronics, 22(1):115-126, Feb 2017. ISSN 1083-4435. doi: 10.1109/TMECH.2016.2618362. URL https://doi.org/10.1109/TMECH.2016.2618362.

[12] J. Veltman, C. Boetes, T. Wobbes, J. G. Blickman, and J. O. Barentsz. Magnetic Resonance-Guided Biopsies and Localizations of the Breast: Initial Experiences Using an Open Breast Coil and Compatible Intervention Device. Investigative Radiology, 40(6), 2005. ISSN 00209996. doi: 10.1097/01.rli.0000163672.90247.70. URL https://doi.org/10.1097/01.rli.0000163672.90247.70.

[13] J. P. Whitney, M. F. Glisson, E. L. Brockmeyer, and J. K. Hodgins. A low-friction passive fluid transmission and fluid-tendon soft actuator. In 2014 IEEE/RSJ 
International Conference on Intelligent Robots and Systems, pages 2801-2808, Sept 2014. doi: 10.1109/IROS. 2014.6942946. URL https://doi.org/10.1109/IROS.2014. 6942946

[14] B. Yang, U. X. Tan, A. B. McMillan, R. Gullapalli, and J. P. Desai. Design and Control of a 1-DOF MRI-Compatible Pneumatically Actuated Robot With Long Transmission Lines. IEEE/ASME Transactions on Mechatronics, 16(6):1040-1048, Dec 2011. ISSN 1083-4435. doi: 10.1109/TMECH.2010.2071393. URL https://doi.org/10.1109/TMECH.2010.2071393. 